\title{
Mean Square Error in ML Estimation of Two-Level Time Series Models
}

\author{
Olasunkanmi Isiaka Azeez \\ Department of Statistics \\ The Federal Polytechnic, \\ Offa, Kwara State, Nigeria
}

\author{
Kunle Bayo Adewoye \\ Department of Statistics \\ The Federal Polytechnic, \\ Offa, Kwara State, Nigeria
}

\begin{abstract}
Two-level time series models are commonly used to analyze longitudinal and correlated data with the standard and parametric assumption that the within-individual (level-1) residuals are uncorrelated rarely checked. There is marked disagreement in the literature as to whether such parametric assumption is important or innocuous. Monte Carlo methods were used to examine the conditions in which the level-1 independence of observations assumption on the parameter estimates of fixed effects was violated and the associated errors due to mean square were investigated. Conditions also varied the series lengths, the numbers of participants per study, and the strength of the autocorrelation coefficient. The simulation results, under the finite sampling properties of Mean Squared Error (MSE), Shown that in finite data, the maximum likelihood estimates may be substantially biased and possess mean square errors substantially higher than Cramer-Rao bounds.
\end{abstract}

\section{Keywords}

Longitudinal, Autocorrelation \& Monte Carlo

\section{INTRODUCTION}

The analysis of change is a fundamental component of so many research endeavours in almost every discipline. Our general idea inferred about change and its measurement, whether the developing traits under study are physiological, morphological, psychological or behavioural, remains a challenging area [1]. Although philosophical discussions of change have been traced back as far as Aristotle [2], various approaches used earlier to investigate change were very limited in that (a) only two occasions of measurement are addressed and (b) they focused exclusively either on measurement occasion level or on subject-level growth, resulting in data gap too scanty to allow examination of some of the most basic and interesting hypotheses about change over time to be tested [3]. Many longitudinal studies are designed to investigate changes over time in a characteristic which is measured repeatedly for each study participant, such as clinical trial in which patients are randomly assigned to different treatments and repeatedly evaluated over the course of the study. When measurements are repeated on the same subjects e.g., animals or students, a 2-level hierarchy is established with measurement repetitions or occasions as level 1 units and subjects as level 2 units. In most cases, the multiple observations are taken over time, but they could be over space, such data are referred to as 'repeated measures' or clustered data. A multilevel problem which concerns a population with a hierarchical structure were designed to analyze repeated measures data and the analysis of such data can be conducted efficiently using a two-level model. price of such a powerful model for treating hierarchically structured data is the requirement of a set of strong mathematical assumptions whose conditions are expected to be violated to some degree in actual studies. As with other statistical techniques, the assumptions of MLM must be valid in order for the estimates and associated significance tests to have the desired properties.

In some cases, especially where measurements are made close together in time, often the error term is not independent through time. Instead, the errors are serially correlated or autocorrelated. If the error term is autocorrelated, the efficiency of ordinary least-squares (OLS) parameter estimates is adversely affected and standard error estimates are biased due to failure to account for the correlated structure of observations [4]. Multilevel models can accommodate nonindependence of observations, a lack of sphericity, missing data, small and/or discrepant group sample sizes, and heterogeneity of variance across repeated measures [5]. As with most statistical models, an important assumption of MLM is that the level-1 errors $\left(e_{i j}\right)$ are independently and normally distributed with a mean of 0 and a variance of $\sigma^{2}$ [6]. Multilevel models are used with repeated measures data to accommodate the fixed effects of covariates and the covariation between observations on the same subject at different times [7]. One of the main reasons we moved to multilevel models rather than just working with linear models was to resolve non-independencies in our data, however, multilevel models can still violate independence. In this paper, we assume data on different subjects are independent, and for simplicity, we assume there are $t$ measurements at the same equally spaced times on each subject.

\section{Inferential Settings}

In ordinary regression analysis, in the case of severe violations, a variety of statistical methods for correcting nonindependence according to Garson (2013) include analysis of variance and other general linear model (GLM) methods that have been adapted to handle non-independence, but these adaptations are problematic. In estimating model parameters when there are random effects, it is necessary to adjust for the covariance structure of the data. The adjustment made by GLM assumes uncorrelated error (that is, it assumes data independence) [8]. Also another method for correcting autocorrelation include modeling the serial correlation explicitly using some error autocorrelation formulation, say an $\mathrm{AR}(1)$ process, and then use the generalized least square (GLS) to estimate the Autocorrelation-Corrected [9].

In multilevel models, specification assumptions apply at each level. Moreover, misspecification at one level can affect 
results at other levels. In most multilevel applications, the errors in the level-1 model are assumed to have equal variance, $\sigma^{2}$. According to [10], if the level-1 variance varies randomly over level-2 units, but these variances are assumed equal, consequences for inference about the level-2 coefficients will be mild, on the other hand if the =variances depend systematically as a function of level-1 or level-2 predictors, consequences may be more serious. Because causes of heterogeneity are quite different in their implications, it is strongly advocated to investigate possible sources of heterogeneity and model it if found.

Finally, it must be emphasized that failure to adequately account for correlation among repeated measures can result in misleading inferences. For instance, if it is assumed that the repeated measures are uncorrelated when in fact there is strong positive correlation, the nominal standard errors (resulting from the naive assumption of independence or uncorrelated repeated measures) will be incorrect [11]. Autocorrelated data are very common for time ordered data, hence, statistical analysis of repeated measures data must address the issue of covariation between measures on the same unit. A key argument being made is that a systematic study investigating the effects of this violation is important and, therefore, addressed in this paper.

The main question to be answered in this paper is, what is the effect of error due to mean square on the efficiency of maximum likelihood (ML) parameters estimates as a result of autocorrelation. Related questions are whether or not the severity of this effect is influenced by the number of measurement occasions, the degree of autocorrelation and the number of subjects. The first two conditions are chosen because when the model includes both random intercepts and slopes (or randomly varying coefficients for any functions of time), the variability of the response can change as a function of the times of measurement, and the magnitudes of the correlations between measurements from the same individual can depend on the time between them.

We ask that authors follow some simple guidelines. In essence, we ask you to make your paper look exactly like this document. The easiest way to do this is simply to download the template, and replace the content with your own material.

\section{MODEL CONCEPTS}

Consider a simple linear regression model for the $\begin{array}{llll}\text { measurement } & Y & \text { of } & \text { individual }\end{array}$ $\mathrm{i}(\mathrm{i}=1,2, \cdots, \mathrm{N}$ subjects $)$ on occasion $\mathrm{j}(\mathrm{j}=$ $1,2, \cdots$ n occasions,

$$
Y_{i j}=\beta_{0}+\beta_{1} X_{i j}+e_{i j}
$$

Ignoring subscripts, this model represents the regression of the outcome variable $Y$ on the independent variable time (denoted $X$ ). The subscripts keep track of the particulars of the data, namely whose observation it is (subscript) and when this observation was made (the subscript $\mathrm{j}$ ). The independent variable $X$ gives a value to the level of time, and may represent time in weeks, months, etc. Since $Y$ and $X$ carry both $i$ and $j$ subscripts, both the outcome variable and the time variable are allowed to vary by individuals and occasions.

In linear reg=ression models, like (1), the errors eij are assumed to be normally and independently distributed in the population with zero mean and common variance $\sigma^{2}$. This independence assumption makes the model given in equation (1) an unreasonable one for repeated measure data. This is because the outcomes $Y$ are observed repeatedly from the same individuals, and so it is much more reasonable to assume that errors within an individual are correlated to some degree. Furthermore, the above model posits that the change across time is the same for all individuals since the model parameters $\left(\beta_{0}\right.$, the intercept or initial level, and $\beta_{1}$, the linear change across time) do not vary by individuals. For both of these reasons, it is useful to add individual-specific effects into the model that will account for the data dependency and describe differential time trends for different individuals. This is precisely what Multilevel Time Series Models for repeated data do.

Estimations of repeated measures data are facilitated by using a multi-level model approach, which allows the estimation of within-individual (level-1) and between-individual (level-2) variations in outcomes. At first, we established a regression equation for the first level variables, in which the tracking results that came from different observation times were the first layer and the invariant individual characteristics were the second layer data.

In the first floor of the data structure, the track observation result was considered as the dependent variable.

$Y_{i j}=\beta_{o j}+\beta_{1 j} X_{i j}+e_{i j}$

In a two-level model each term has two subscripts, the first of which corresponds to level 1 while the second refers to level 2. As in "(2)", subscript "0" means intercept, subscript "1" means slope, subscript " $i$ " means the $i$-th observation object, Subscript " $j$ " indicates the $j$-th observation time.

" $\beta_{o j} "$ is the intercept of the equation, it indicates the average of the $i$ - $t h$ observed objects.

" $\beta_{1 j}$ " is the regression coefficient, it indicates the changing rate of the $i-t h$ observation object.

" $X_{i j}$ " means the values of the variable $X$ when the $i-t h$ observed object is in the $j-t h$ observation time.

" $e_{i j} "$ means residual, the implication is that the measured value $Y$ of the $i$ - $t h$ object in the $j-t h$ observation time that cannot be explained by the independent variable $X$.

Equation (2) is similar to the general regression equation, the only difference is, intercept and slope are not constant.

In the second layer of the data structures, the intercept and slope are used as the dependent variable in (2), and individual characteristics are considered as independent variables, the we create two regression equations for the second layer:

$$
\begin{gathered}
\left\{\begin{array}{l}
\beta_{o j}=\gamma_{00}+\gamma_{01} W_{j}+\mathrm{u}_{0 j} \\
\beta_{1 j}=\gamma_{10}+\gamma_{11} W_{j}+\mathrm{u}_{1 j}
\end{array}\right. \\
\text { where }\left\{\begin{array}{c}
Y_{i j}=\beta_{0 j}+e_{i j} \\
\beta_{o j}=\gamma_{00}+u_{0 j}
\end{array}\right.
\end{gathered}
$$

is referred to as the null model.

In equations (3) and (4), each parameter has two subscripts, if 
the first subscripts is " 0 ", this is the parameter that relates to the intercept of (2). if the first subscript is "1", this is the parameter that relates to the slope of (2). if the second subscripts is " 0 ", it means the intercept part of the second layer equation, if the second subscript is "1", it means the slope part of the second layer equation.

$\gamma_{00}$ is the intercept of (3), it can be understood as the average of the dependent variable $\mathrm{Y}$ when the independent variable $W_{j}$ is 0 .

$W_{j}$ is the value on the level-2 predictor

$\gamma_{01}$ is the regression coefficients of the variables $W_{j}$ in (3), it can be understood as the impact of the variable $W_{j}$ to the initial value of the dependent variable $Y$.

$\gamma_{10}$ is the intercept of (4), it can be understood as the changing rate of observed object when the variable $W_{j}$ is 0 .

$\gamma_{11}$ is the regression coefficient of the variable $W_{j}$ in (4), it can be understood as the effect of the variable $W_{j}$ on the changing rate.

$u_{0 j}$ is the residual of (3), is the intercept deviation for subject $i$, it represents the influence of individual $i$ on his or her repeated observations.

$u_{1 j}$ is the residual of (4) is the slope deviation for subject $i$

The assumption regarding the independence of the errors is one of conditional independence, that is, they are independent conditional on $u_{0 j}$ and $u_{1 j}$.

Our model (2) with one time-level and one individual level explanatory variable can be written as a single complex multilevel time series regression equation by

Substituting $\quad \gamma_{00}+\gamma_{01} W_{j}+\mathrm{u}_{0 j}$ for $\beta_{0 j}$, and Substituting $\gamma_{10}+\gamma_{11} W_{j}+\mathrm{u}_{1 j}$ for $\beta_{1 j}$, and redistributing, we have:

$Y_{i j}=\gamma_{00}+\gamma_{01} W_{j}+\mathrm{u}_{0 j}+\gamma_{10} X_{i j}+$ $\gamma_{11} W_{j} X_{i j}+\mathrm{u}_{1 j} X_{i j}+e_{i j}$

Rearranging, so that the fixed effects appear first, followed by the random effects, leads us to our final mixed model, defined as

$Y_{i j}=\gamma_{00}+\gamma_{10} X_{i j}+\gamma_{01} W_{j}+\gamma_{11} X_{i j} W_{j}+$ $u_{1 j} X_{i j}+u_{0 j}+e_{i j}$

[13] remarks that " In order to allow for the classification of variables and coefficients in terms of the level of hierarchy they affect, a combined model is created by rearranging so that the fixed effects appear first, followed by the random effects.

The term $X_{i j} W_{j}$ is an interaction term that appears in the model because of modeling the varying regression slope $\beta_{1 j}$ of the time-level variable $X_{i j}$ with the individual level variable $W_{j}$.

In equation (7), the errors are no longer independent across the level units. The terms $u_{0 j}$ and $u_{1 j}$ demonstrate that there is dependency among the level-1 units nested within each level-2 unit. Furthermore, $u_{0 j}$ and $u_{1 j}$ may have different values within level-2 units, leading to heterogeneous variances of the error terms [6]. That is (7) shows that the composite error structure, $u_{1 j} X_{i j}+u_{0 j}+e_{i j}$ is now clearly heteroscedastic since it is conditioned on level of the explanatory variable.

\section{METHODS}

The simulation model and procedure

We use two different simple two-level models, with one explanatory variable each at the individual level and one explanatory variable at the subject level, conforming to equation (7) above. The model used in the process of generating data for the present study is the first model shown below with $\mathrm{W}$ replaced by Z, which henceforth will be referred to as autocorrelated model.

$$
\begin{aligned}
& \text { Level-1: } Y_{i j}=\beta_{o j}+\beta_{1 j} X_{i j}+e_{i j} \\
& \text { Level-2: } \beta_{0 j}=\beta_{0}+\beta_{01} Z_{j}+\mathrm{u}_{0 j} \\
& \qquad \beta_{1 j}=\beta_{1}+\beta_{11} Z_{j}+\mathrm{u}_{1 j} \\
& e_{i j} \sim N\left(0, \sigma^{2} \Omega_{\mathrm{k}}\right) . \\
& \text { Thus, } V\left(y_{i}\right)=Z_{i} \Sigma_{v} Z_{i}{ }^{\prime}+\sigma^{2} \Omega_{\mathrm{k}}
\end{aligned}
$$

where $\Omega_{\mathrm{k}}$ depends on $\mathrm{q}$ autocorrelation parameters, with $\mathrm{q}$ varying depending on the type of autocorrelated error structure being considered.

The motivation lies in the need to allow for patterns of dependence, rather than complete independence among response values. The simplest way to allow such dependence is to assume $V\left(y_{i}\right)=Z_{i} \Sigma_{v} Z_{i}{ }^{\prime}+\sigma^{2} \Omega_{\mathrm{k}}$ with $\Omega_{\mathrm{k}}$ of dimension $N \times N$, symmetric and positive definite or semi positive definite (which allows any covariance matrix).

The second model is given below, which will henceforth be referred to as standard model.

$$
\begin{array}{ll}
\text { Level-1: } Y_{i j}=\beta_{o j}+\beta_{1 j} X_{i j}+e_{i j} & \text { (2 repeated) } \\
\text { Level-2: } \beta_{0 j}=\beta_{0}+\beta_{01} Z_{j}+\mathrm{u}_{0 j} & \text { (8 repeated) } \\
\beta_{1 j}=\beta_{1}+\beta_{11} Z_{j}+\mathrm{u}_{1 j} & \text { (9 repeated) } \\
e_{i} \sim N\left(0, \sigma^{2} \mathrm{I}\right) & \\
\text { Thus, } V\left(y_{i}\right)=Z_{i} \Sigma_{v} Z_{i}^{\prime}+\sigma^{2} \mathrm{I} & \text { (11) }
\end{array}
$$

\section{Estimation methods for variance components}

For the purpose of this study, used were made of $\mathrm{R}$ program to estimate the parameters. The estimation methods are compared in relation to the number of subjects, number of measurement occasion, and autocorrelation coefficient under 
the following conditions:

I. autocorrelation coefficients of $0.3,0.7,0.99$

II. variances of intercept and slopes and their covariances of $-12.63,2.08$ and -1.42 respectively

III. numbers of subjects - 30, 50, and 100

IV. numbers of observation within subjects $-3,5$, and 10

V. 1000 replication for each condition

For the regression coefficients, 1.00 was chosen for the intercept, and 0.3 for all the regression slopes (medium effect size; see [13]; [14]. The first level variance $\sigma^{2}$ was fixed at 12.22 , while the error terms in the simulated data are auto regressively correlated. The sizes of the conditions are partially based on literature and partially on practical experience.

\section{RESULTS}

\subsection{Coverage}

In order to investigate the influence of the number of subjects, the autocorrelation coefficients and the number of measurement occasions on the estimation of error due to mean square on the parameter estimates, the coverage per condition was calculated to describes the uncertainty inherent in our estimate, and describes a range of values within which we can be reasonably sure that the true effect actually lies. The results are as shown below.

Table 1: Full Maximum Likelihood Parameter Estimates and Wald 95\% Confidence Intervals (CIs) Obtained by Fitting Model with Autocorrelated Errors.

\begin{tabular}{|l|l|l|l|l|l|}
\hline ML/COMB & $\begin{array}{l}\text { ML SLOPES } \\
\text { (Estimate) }\end{array}$ & VAR. SLOPES & STD DEV SLOPES & $\begin{array}{l}\text { 95\% CI } \\
\text { (LOWER) }\end{array}$ & $\begin{array}{l}\text { 95\% CI } \\
\text { (UPPER) }\end{array}$ \\
\hline $3,0.3,30$ & -0.3267 & 2.2146 & 1.4882 & -3.2434 & 2.5901 \\
\hline $5,0.3,30$ & -0.2107 & 2.1143 & 1.4541 & -3.0606 & 2.6393 \\
\hline $10,0.3,30$ & 0.0331 & 2.0855 & 1.4441 & -2.7974 & 2.8636 \\
\hline $3,0.7,30$ & -0.4429 & 2.1283 & 1.4589 & -3.3022 & 2.4165 \\
\hline $5,0.7,30$ & -0.3591 & 2.0942 & 1.4472 & -3.1955 & 2.4773 \\
\hline $10,0.7,30$ & 0.3363 & 2.0830 & 1.4433 & -2.4925 & 3.1651 \\
\hline $3,0.9,30$ & -0.4745 & 2.0946 & 1.4473 & -3.3111 & 2.3622 \\
\hline $5,0.9,30$ & -0.3867 & 2.0844 & 1.4437 & -3.2164 & 2.4431 \\
\hline $10,0.9,30$ & 0.4070 & 2.0810 & 1.4426 & -2.4205 & 3.2344 \\
\hline $3,0.3,50$ & 0.6721 & 2.1605 & 1.4698 & -2.20879 & 3.5530 \\
\hline $5,0.3,50$ & 0.4776 & 2.1005 & 1.4493 & -2.3630 & 3.3183 \\
\hline $10,0.3,50$ & 1.2309 & 2.0833 & 1.4434 & -1.5981 & 4.0599 \\
\hline $3,0.7,50$ & 0.7294 & 2.1088 & 1.4522 & -2.1168 & 3.5757 \\
\hline $5,0.7,50$ & 0.6169 & 2.0885 & 1.4451 & -2.2156 & 3.4494 \\
\hline $10,0.7,50$ & 1.6538 & 2.0818 & 1.4428 & -1.1742 & 4.4818 \\
\hline $3,0.9,50$ & 0.7547 & 2.0887 & 1.4452 & -2.0779 & 3.5873 \\
\hline $5,0.9,50$ & 0.6361 & 2.0826 & 1.4431 & -2.1925 & 3.4646 \\
\hline $10,0.9,50$ & 1.7345 & 2.0806 & 1.4424 & -1.0927 & 4.5616 \\
\hline $3,0.3,100$ & 0.8482 & 2.1201 & 1.4561 & -2.0057 & 3.7021 \\
\hline $5,0.3,100$ & 1.1363 & 2.0902 & 1.4458 & -1.6974 & 3.9700 \\
\hline $10,0.3,100$ & 0.5546 & 2.0817 & 1.4428 & -2.2733 & 3.3824 \\
\hline $3,0.7,100$ & 0.9212 & 2.0943 & 1.4472 & -1.9153 & 3.7577 \\
\hline $5,0.7,100$ & 1.2759 & 2.0842 & 1.4437 & -1.5537 & 4.1055 \\
\hline $10,0.7,100$ & 0.6573 & 2.0809 & 1.4425 & -2.1701 & 3.4846 \\
\hline $3,0.9,100$ & 0.9541 & 2.0843 & 1.4437 & -1.8756 & 3.7838 \\
\hline $5,0.9,100$ & 1.2959 & 2.0813 & 1.4427 & -1.5318 & 4.1235 \\
\hline $10,0.9,100$ & 0.6758 & 2.0803 & 1.4423 & -2.1512 & 3.5027 \\
\hline
\end{tabular}




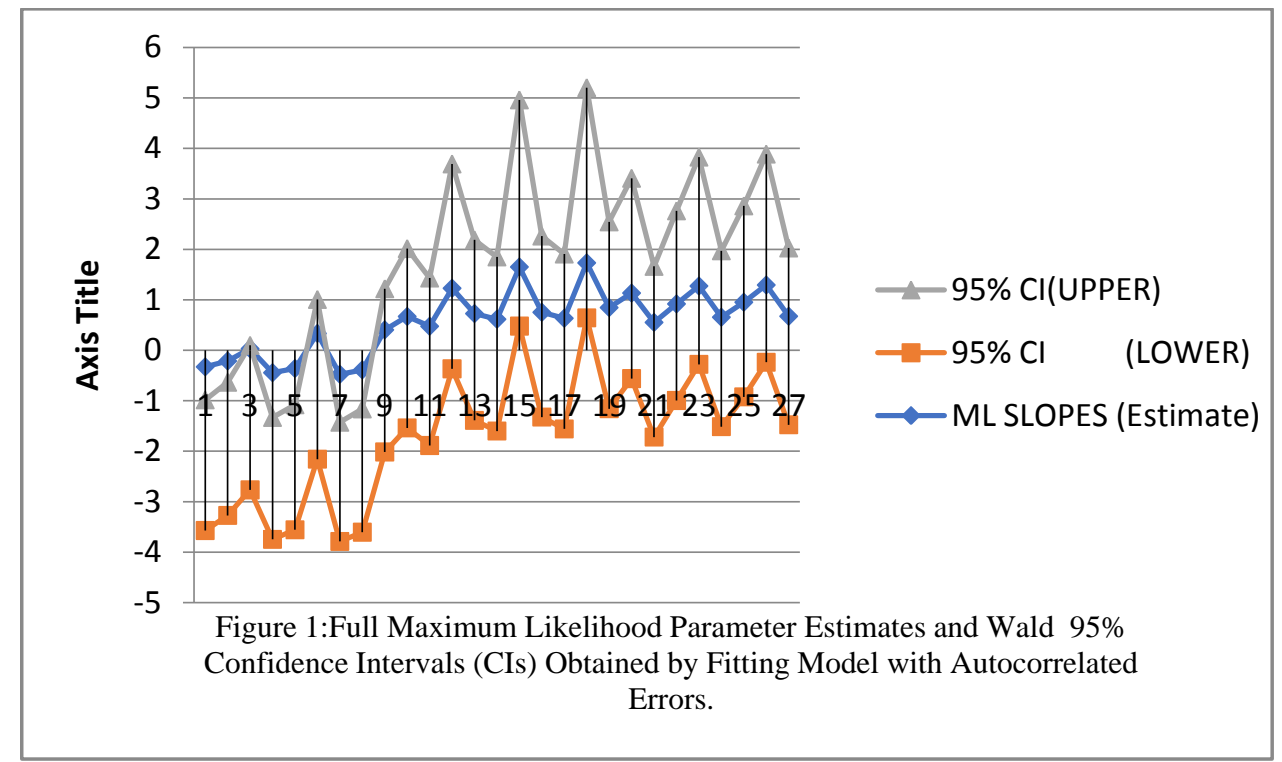

Table 2: Restricted Maximum Likelihood Parameter Estimates and Wald 95\% Confidence Intervals (CIs) Obtained by Fitting Model with Autocorrelated Errors.

\begin{tabular}{|l|l|l|l|l|l|}
\hline REML/COMB & $\begin{array}{l}\text { REML SLOPES } \\
\text { (Estimate) }\end{array}$ & VAR. SLOPES & STD DEV SLOPES & $\begin{array}{l}\text { 95\% CI } \\
\text { (LOWER) }\end{array}$ & $\begin{array}{l}\text { 95\% CI } \\
\text { (UPPER) }\end{array}$ \\
\hline $3,0.3,30$ & -0.3172 & 2.2353 & 1.4951 & -3.2475 & 2.6132 \\
\hline $5,0.3,30$ & -0.2183 & 2.1229 & 1.4570 & -3.0741 & 2.6374 \\
\hline $10,0.3,30$ & 0.0301 & 2.0887 & 1.4453 & -2.8025 & 2.8628 \\
\hline $3,0.7,30$ & -0.4328 & 2.1357 & 1.4614 & -3.2971 & 2.4316 \\
\hline $5,0.7,30$ & -0.3653 & 2.0978 & 1.4484 & -3.2042 & 2.4735 \\
\hline $10,0.7,30$ & 0.3314 & 2.0848 & 1.4439 & -2.4986 & 3.1614 \\
\hline $3,0.9,30$ & -0.4642 & 2.0968 & 1.4480 & -3.3024 & 2.3740 \\
\hline $5,0.9,30$ & -0.3928 & 2.0855 & 1.4441 & -3.2233 & 2.4377 \\
\hline $10,0.9,30$ & 0.4016 & 2.0815 & 1.4428 & -2.4262 & 3.2294 \\
\hline $3,0.3,50$ & 0.6838 & 2.1674 & 1.4722 & -2.2018 & 3.5693 \\
\hline $5,0.3,50$ & 0.4649 & 2.1033 & 1.4503 & -2.3776 & 3.3075 \\
\hline $10,0.3,50$ & 1.2259 & 2.0842 & 1.4437 & -1.6037 & 4.0556 \\
\hline $3,0.7,50$ & 0.7407 & 2.1113 & 1.4530 & -2.1072 & 3.5886 \\
\hline $5,0.7,50$ & 0.6050 & 2.0896 & 1.4456 & -2.2283 & 3.4383 \\
\hline $10,0.7,50$ & 1.6475 & 2.0823 & 1.4430 & -1.1808 & 4.4758 \\
\hline $3,0.9,50$ & 0.7658 & 2.0894 & 1.4455 & -2.0673 & 3.5990 \\
\hline $5,0.9,50$ & 0.6244 & 2.0830 & 1.4432 & -2.2044 & 3.4531 \\
\hline $10,0.9,50$ & 1.7278 & 2.08074 & 1.4425 & -1.0994 & 4.5551 \\
\hline $3,0.3,100$ & 0.8575 & 2.1218 & 1.4566 & -1.9975 & 3.7125 \\
\hline $5,0.3,100$ & 1.1347 & 2.0909 & 1.4460 & -1.6994 & 3.9689 \\
\hline $10,0.3,100$ & 0.5519 & 2.0819 & 1.4429 & -2.2761 & 3.3799 \\
\hline $3,0.7,100$ & 0.9304 & 2.0949 & 1.4474 & -1.9064 & 3.7673 \\
\hline $5,0.7,100$ & 1.2734 & 2.0845 & 1.4438 & -1.5564 & 4.1032 \\
\hline $10,0.7,100$ & 0.6531 & 2.0810 & 1.4426 & -2.1744 & 3.4805 \\
\hline $3,0.9,100$ & 0.9633 & 2.0845 & 1.4438 & -1.8665 & 3.7931 \\
\hline $5,0.9,100$ & 1.2931 & 2.0814 & 1.4427 & -1.5345 & 4.1208 \\
\hline $10,0.9,100$ & 0.67133 & 2.0803 & 1.4423 & -2.15569 & 3.4982 \\
\hline & & & & \\
\hline
\end{tabular}




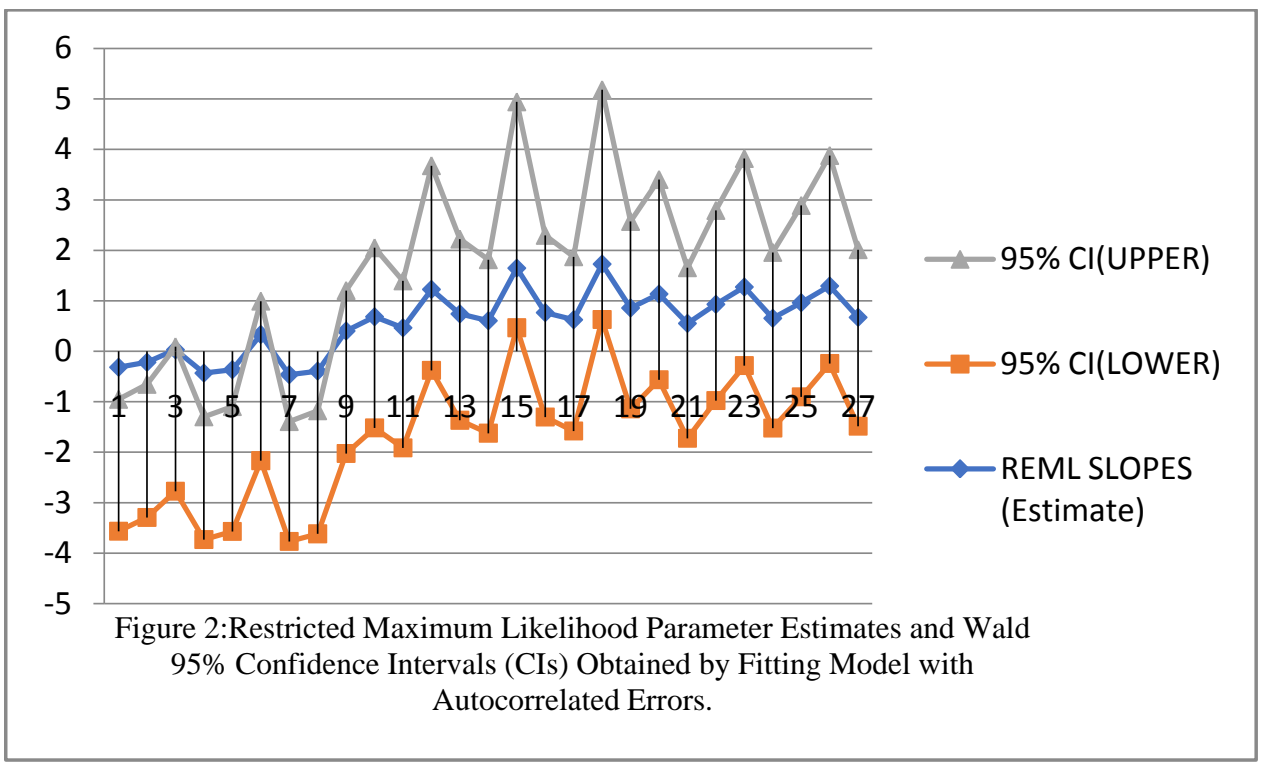

Table 3: Full Maximum Likelihood Parameter Estimates and Wald 95\% Confidence Intervals (CIs) Obtained by Fitting Standard Multilevel Model.

\begin{tabular}{|l|l|l|l|l|l|}
\hline MLI/COMB & $\begin{array}{l}\text { MLI SLOPES } \\
\text { (Estimate) }\end{array}$ & VAR. SLOPES & STD DEV SLOPES & $\begin{array}{l}\text { 95\% CI } \\
\text { (LOWER) }\end{array}$ & $\begin{array}{l}\text { 95\% CI } \\
\text { (UPPER) }\end{array}$ \\
\hline $3,0.3,30$ & -0.1629 & 2.2837 & 1.5112 & -3.1248 & 2.7990 \\
\hline $5,0.3,30$ & -0.0521 & 2.1207 & 1.4563 & -2.9064 & 2.8022 \\
\hline $10,0.3,30$ & -0.1884 & 2.0849 & 1.4439 & -3.0185 & 2.6417 \\
\hline $3,0.7,30$ & -0.1106 & 2.2837 & 1.5112 & -3.0725 & 2.8513 \\
\hline $5,0.7,30$ & -0.0670 & 2.1207 & 1.4563 & -2.9213 & 2.7873 \\
\hline $10,0.7,30$ & -0.1770 & 2.0849 & 1.4439 & -3.0071 & 2.6531 \\
\hline $3,0.9,30$ & -0.0844 & 2.2837 & 1.5112 & -3.0463 & 2.8775 \\
\hline $5,0.9,30$ & -0.0745 & 2.1207 & 1.4563 & -2.9288 & 2.7798 \\
\hline $10,0.9,30$ & -0.1712 & 2.0849 & 1.4439 & -2.0013 & 2.6589 \\
\hline $3,0.3,50$ & 0.6542 & 2.2022 & 1.4840 & -2.2544 & 3.5628 \\
\hline $5,0.3,50$ & 0.2743 & 2.1044 & 1.4507 & -2.5690 & 3.1176 \\
\hline $10,0.3,50$ & 0.8867 & 2.0830 & 1.4432 & -1.9421 & 3.7154 \\
\hline $3,0.7,50$ & 0.6775 & 2.2022 & 1.4840 & -2.2311 & 3.5861 \\
\hline $5,0.7,50$ & 0.2710 & 2.1044 & 1.45067 & -2.5723 & 3.1143 \\
\hline $10,0.7,50$ & 0.8901 & 2.0830 & 1.4432 & -1.9387 & 3.7188 \\
\hline $3,0.9,50$ & 0.6891 & 2.2022 & 1.4840 & -2.2195 & 3.5977 \\
\hline $5,0.9,50$ & 0.2694 & 2.1044 & 1.4507 & -2.5739 & 3.1127 \\
\hline $10,0.9,50$ & 0.8918 & 2.0830 & 1.4432 & -1.9370 & 3.7205 \\
\hline $3,0.3,100$ & 0.8299 & 2.1411 & 1.4632 & -2.0380 & 3.6979 \\
\hline $5,0.3,100$ & 0.9397 & 2.0922 & 1.4465 & -1.8953 & 3.7748 \\
\hline $10,0.3,100$ & 0.4663 & 2.0815 & 1.4427 & -2.3615 & 3.29407 \\
\hline $3,0.7,100$ & 0.8781 & 2.1411 & 1.4632 & -1.9899 & 3.7460 \\
\hline $5,0.7,100$ & 0.9402 & 2.0922 & 1.4465 & -1.8948 & 3.7752 \\
\hline $10,0.7,100$ & 0.4656 & 2.0815 & 1.4427 & -2.3622 & 3.2934 \\
\hline $3,0.9,100$ & 0.90214 & 2.1411 & 1.4632 & -1.9658 & 3.7701 \\
\hline $5,0.9,100$ & 0.9404 & 2.0922 & 1.4465 & -1.8946 & 3.7755 \\
\hline $10,0.9,100$ & 0.4652 & 2.0815 & 1.4427 & -2.3625 & 3.29301 \\
\hline & & & & \\
\hline
\end{tabular}




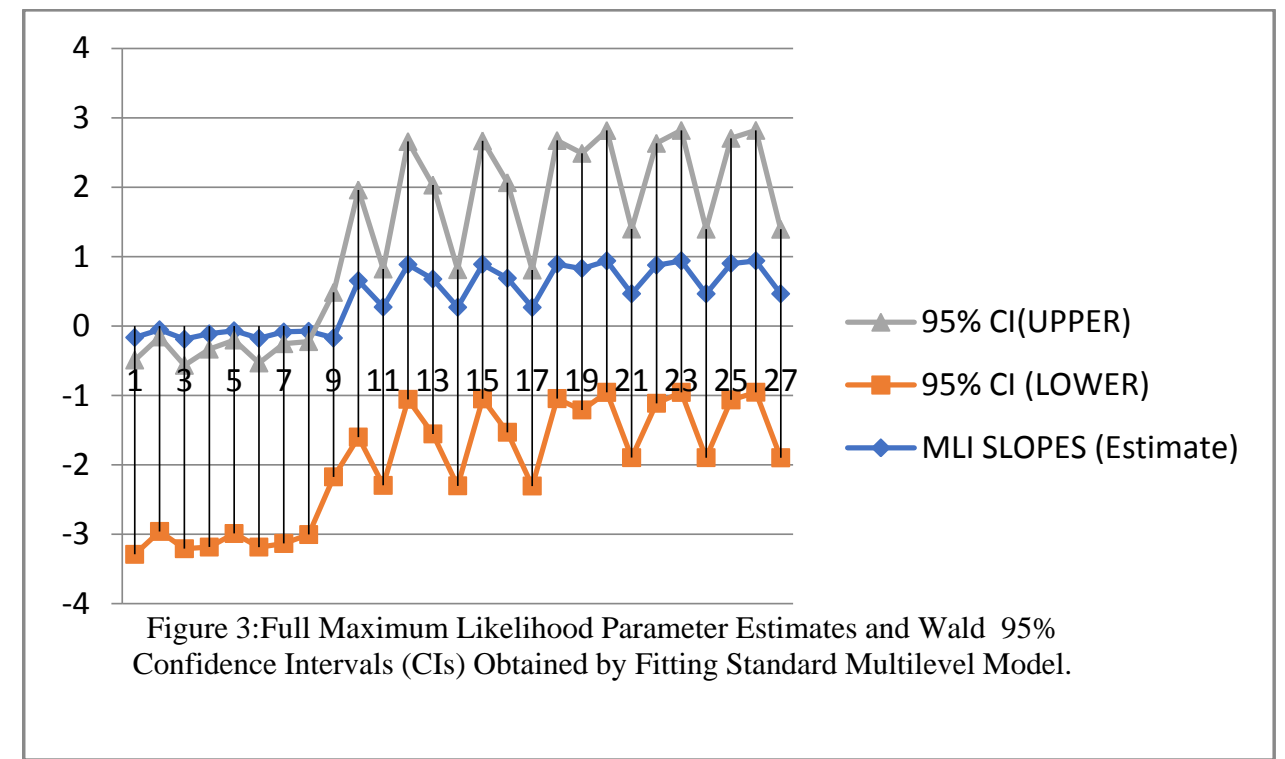

Table 4: Restricted Maximum Likelihood Parameter Estimates and Wald 95\% Confidence Intervals (CIs) Obtained by Fitting Standard Multilevel Model.

\begin{tabular}{|l|l|l|l|l|l|}
\hline REMLI/COMB & $\begin{array}{l}\text { REMLI SLOPES } \\
\text { (Estimate) }\end{array}$ & VAR. SLOPES & STD DEV SLOPES & $\begin{array}{l}\text { 95\% CI } \\
\text { (LOWER) }\end{array}$ & $\begin{array}{l}\text { 95\% CI } \\
\text { (UPPER) }\end{array}$ \\
\hline $3,0.3,30$ & -0.1257 & 2.3150 & 1.5215 & -3.1079 & 2.8564 \\
\hline $5,0.3,30$ & -0.0414 & 2.1309 & 1.4598 & -2.9026 & 2.8197 \\
\hline $10,0.3,30$ & -0.1930 & 2.0878 & 1.4449 & -3.0251 & 2.6390 \\
\hline $3,0.7,30$ & -0.0734 & 2.3150 & 1.5215 & -3.0555 & 2.9088 \\
\hline $5,0.7,30$ & -0.0563 & 2.1309 & 1.4598 & -2.9175 & 2.8048 \\
\hline $10,0.7,30$ & -0.1816 & 2.0878 & 1.4449 & -3.0136 & 2.6505 \\
\hline $3,0.9,30$ & -0.0472 & 2.3150 & 1.5215 & -3.0294 & 2.9350 \\
\hline $5,0.9,30$ & -0.0638 & 2.1309 & 1.4598 & -2.9249 & 2.7974 \\
\hline $10,0.9,30$ & -0.1759 & 2.0878 & 1.4449 & -3.0079 & 2.6562 \\
\hline $3,0.3,50$ & 0.6578 & 2.2128 & 1.4876 & -2.2578 & 3.5734 \\
\hline $5,0.3,50$ & 0.2707 & 2.1078 & 1.4518 & -2.5749 & 3.1162 \\
\hline $10,0.3,50$ & 0.8855 & 2.0838 & 1.4435 & -1.9439 & 3.7148 \\
\hline $3,0.7,50$ & 0.6811 & 2.2128 & 1.4876 & -2.2345 & 3.5967 \\
\hline $5,0.7,50$ & 0.2674 & 2.1078 & 1.4518 & -2.5782 & 3.1129 \\
\hline $10,0.7,50$ & 0.8889 & 2.0838 & 1.4435 & -1.9405 & 3.7182 \\
\hline $3,0.9,50$ & 0.6927 & 2.2128 & 1.4876 & -2.2229 & 3.6083 \\
\hline $5,0.9,50$ & 0.2657 & 2.1078 & 1.4518 & -2.5799 & 3.1113 \\
\hline $10,0.9,50$ & 0.8906 & 2.0838 & 1.4435 & -1.9388 & 3.7199 \\
\hline $3,0.3,100$ & 0.8229 & 2.1436 & 1.4641 & -2.0468 & 3.69257 \\
\hline $5,0.3,100$ & 0.9326 & 2.0930 & 1.4467 & -1.9029 & 3.76821 \\
\hline $10,0.3,100$ & 0.4671 & 2.08167 & 1.4428 & -2.3608 & 3.2950 \\
\hline $3,0.7,100$ & 0.8710 & 2.1436 & 1.4641 & -1.9986 & 3.7407 \\
\hline $5,0.7,100$ & 0.9331 & 2.0930 & 1.4467 & -1.9025 & 3.7687 \\
\hline $10,0.7,100$ & 0.4664 & 2.0817 & 1.4428 & -2.3615 & 3.2943 \\
\hline $3,0.9,100$ & 0.8951 & 2.1436 & 1.4641 & -1.9746 & 3.7648 \\
\hline $5,0.9,100$ & 0.9334 & 2.0930 & 1.4467 & -1.9022 & 3.7689 \\
\hline $10,0.9,100$ & 0.4660 & 2.0817 & 1.4428 & -2.3619 & 3.2939 \\
\hline
\end{tabular}




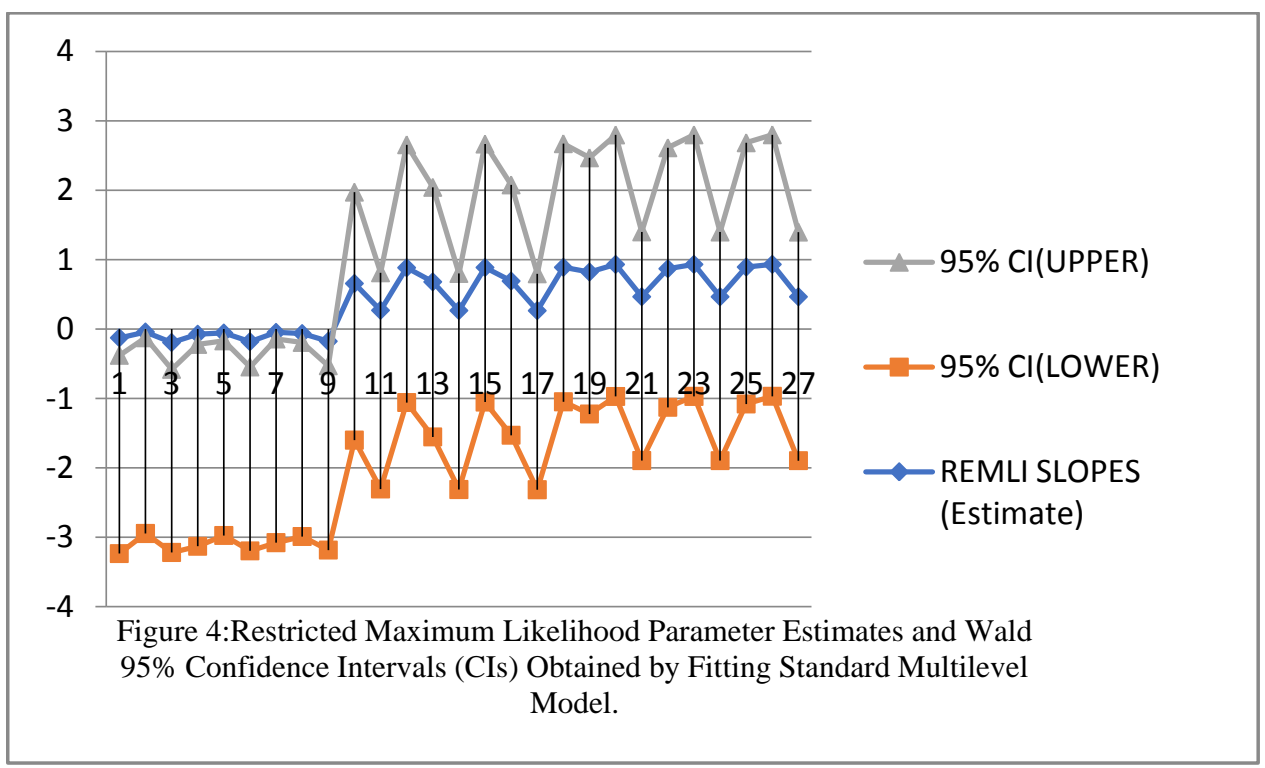

Observations: Our goal here is to just get a sense for the data: What do the trajectories look like? Supposing we fit individual regressions, how do the regression coefficients vary across subjects? Because the slope varies for individuals, the fitted model allows the possibility that some individuals do not change across time, while others can exhibit dramatic change. zero is within all of the prediction intervals on the random effects, that the interval we have constructed includes zero reflects the fact that there is considerable heterogeneity in terms of subjects change across time. By the value of the dispersion of the coefficients, some subjects are above the line of the overall average slope while others are below it, the variation around the average line (as indicated by the value of variances) is large, this implies that individuals' slopes are not similar and cannot be modeled by the fixed-effect estimate.

Wald simplest $95 \%$ confidence intervals (CI) on the estimated average slopes were constructed, by taking the point estimate \pm 1.96 estimated standard errors in order to determine lopsidedness of coverage resulting from errors due to mean square and find the influence of the number of subjects, the autocorrelation coefficient and the number of measurement occasions on the constructed CI for the parameter estimates. More specifically, when using the Wald Confidence Interval, two points on either side of MLE are chosen such that they are equidistant from MLE value (MLE $\pm \mathrm{SE} *$ (1-alpha)/2 percentile of Normal distribution).

The width of REML confidence interval estimates are wider than the ML confidence interval, but the differences are small. In standard multi-level model, combinations with same numbers of subjects and measurements occasion appear to have the same width as their prediction interval, this adds quite a bit to our understanding of the variability in our random coefficients. Also observed from the constructed confidence interval, there is lopsidedness of coverage resulting in estimates falling more frequently to one side than the other of the true parameter.

To substantiate our claim, standard normal distribution was used to estimate the expected percentage of regression coefficients that are less than 0.3 under autocorrelated model $(\mathrm{ML} \Omega)$, and found to be within the range of $0.15 \%$ to $100 \%$.

Lopsidedness of coverage is a direct consequence of the bias in the multilevel point estimator, on which the Wald interval is centered. Despite this problem, multilevel Wald 95\% intervals appear to provide conservatively valid (i.e. at least 95\%) average coverage for the parameter estimates (Sander, 2000).

In our constructed $95 \%$ confidence interval, subjects (level-2 units) with low numbers of measurement occasion and low autocorrelation coefficients are predicted to have a wider confidence interval than subjects with high numbers of measurement occasion and high autocorrelation coefficient. Similarly, differences between the number of subjects indicate relationship between the width of the confidence interval. Not surprising, intercept and slope coefficients are random variables that vary across subjects, the specific values for the intercept and slope coefficients are subjects characteristics.

\subsection{Evaluating Mean Square Error}

For the assessment of the parameter estimates, the mean square due to error for each parameter was considered and the results are as shown below;

Table 5: Comparing the MSE of slopes for measurement occasion, $t=3$ for two estimators under standard and correlated models, for different numbers of subject $N$ and different autocorrelation coefficient as a function of the slope.

\begin{tabular}{|l|l|l|l|l|l|l|l|l|l|l|l|l|}
\hline & \multicolumn{3}{|c|}{ ML $(\Omega)$} & \multicolumn{3}{c|}{ REML $(\Omega)$} & \multicolumn{3}{c|}{ ML $(I)$} & \multicolumn{3}{c|}{ REML $(I)$} \\
\hline $\mathbf{N}$ & \multicolumn{3}{|c|}{$\rho$} & \multicolumn{3}{c|}{$\rho$} & $\rho$ & \multicolumn{3}{c|}{$\rho$} \\
\hline & 0.3 & 0.7 & 0.9 & 0.3 & 0.7 & 0.9 & 0.3 & 0.7 & 0.9 & 0.3 & 0.7 & 0.9 \\
\hline 30 & 0.4082 & 0.5675 & 0.6155 & 0.3983 & 0.5546 & 0.6018 & 0.2330 & 0.1873 & 0.1665 & 0.1975 & 0.1556 & 0.1368 \\
\hline 50 & 0.1484 & 0.1945 & 0.2168 & 0.1593 & 0.2063 & 0.2291 & 0.1335 & 0.1505 & 0.1594 & 0.1372 & 0.1544 & 0.1634 \\
\hline 100 & 0.3067 & 0.3921 & 0.4341 & 0.3143 & 0.4010 & 0.4436 & 0.2851 & 0.3385 & 0.3669 & 0.2786 & 0.3313 & 0.3594 \\
\hline
\end{tabular}

Where $\Omega$ represents autocorrelation matrix and $I$ is the identity matrix. 
Table 6: Comparing the MSE of slopes for measurement occasion, $t=5$ for two estimators under standard and correlated models, for different numbers of subject $N$ and different autocorrelation coefficient as a function of the slope.

\begin{tabular}{|c|c|c|c|c|c|c|c|c|c|c|c|c|}
\hline & \multicolumn{3}{|c|}{ ML $(\Omega)$} & \multicolumn{3}{|c|}{$\operatorname{REML~}(\Omega)$} & \multicolumn{3}{|c|}{ ML $(I)$} & \multicolumn{3}{|c|}{ REML (I) } \\
\hline \multirow{2}{*}{$\mathbf{N}$} & \multicolumn{3}{|l|}{$\rho$} & \multicolumn{3}{|c|}{$\rho$} & \multicolumn{3}{|l|}{$\rho$} & \multicolumn{3}{|l|}{$\rho$} \\
\hline & 0.3 & 0.7 & 0.9 & 0.3 & 0.7 & 0.9 & 0.3 & 0.7 & 0.9 & 0.3 & 0.7 & 0.9 \\
\hline 30 & 0.2640 & 0.4381 & 0.4753 & 0.2735 & 0.4483 & 0.4857 & 0.1273 & 0.1381 & 0.1436 & 0.1191 & 0.1295 & 0.1349 \\
\hline 50 & 0.0345 & 0.1037 & 0.1163 & 0.0293 & 0.0955 & 0.1077 & 0.0026 & 0.0028 & 0.0029 & 0.0033 & 0.0035 & 0.0036 \\
\hline 100 & 0.7005 & 0.9537 & 0.9930 & 0.6978 & 0.9486 & 0.9875 & 0.4103 & 0.4109 & 0.4112 & 0.4014 & 0.4020 & 0.4023 \\
\hline
\end{tabular}

Where $\Omega$ represents autocorrelation matrix and $I$ is the identity matrix.

Table 7: Comparing the MSE of slopes for measurement occasion, $t=10$ for two estimators under standard and correlated models, for different numbers of subject $\mathrm{N}$ and different autocorrelation coefficient as a function of the slope.

\begin{tabular}{|c|c|c|c|c|c|c|c|c|c|c|c|c|}
\hline & \multicolumn{3}{|l|}{ ML $(\Omega)$} & \multicolumn{3}{|c|}{$\operatorname{REML}(\Omega)$} & \multicolumn{3}{|l|}{ ML (I) } & \multicolumn{3}{|c|}{ REML (I) } \\
\hline $\mathbf{N}$ & $\rho$ & & & $\rho$ & & & $\rho$ & & & $\rho$ & & \\
\hline & 0.3 & 0.7 & 0.9 & 0.3 & 0.7 & 0.9 & 0.3 & 0.7 & 0.9 & 0.3 & 0.7 & 0.9 \\
\hline 30 & 0.0717 & 0.0021 & 0.0123 & 0.0733 & 0.0017 & 0.0112 & 0.2390 & 0.2279 & 0.2225 & 0.2434 & 0.2323 & 0.2268 \\
\hline 50 & 0.8668 & 1.8333 & 2.0583 & 0.8576 & 1.8161 & 2.0391 & 0.3444 & 0.3484 & 0.3504 & 0.3430 & 0.3470 & 0.3490 \\
\hline 100 & 0.0649 & 0.1279 & 0.1414 & 0.0636 & 0.1249 & 0.1381 & 0.0278 & 0.0275 & 0.0274 & 0.0280 & 0.0278 & 0.0277 \\
\hline
\end{tabular}

\section{Comment:}

As can be seen in Table 5 to Table 7, The violation of the first-level residual independence assumption has a major effect, as expected, on MSE of the estimates of the fixed effect. From the illustrating Tables 5-7, it was noticed that when the model was correctly specified (C-Model) the MSE for the fixed effect increased substantially (between 0.0021 and 2.0583 for FML and between 0.0017 and 2.0391 for REML for slope). Similarly, by omitting correlation matrix (i.e., misspecified model), the MSE for the fixed effect increased substantially (between 0.0026 and 0.4112 for FML, and between 0.0033 and 0.4023 for REML respectively). For the C-Model, lowest MSE occurs at $\rho=0.7$, while the highest occurs at $\rho=0.9$ similarly, for S-Model, $\rho=0.3$ for the lowest and 0.9 for the highest.

In general, increasing autocorrelation level led to an increase in the MSE of the fixed effect estimates of the slope under correctly specified model except when $\mathrm{N}=30$ which is not clearly defined. Similarly, increasing autocorrelation level led to an increase in the MSE of the fixed effect estimates of the slope under misspecified model when $\mathrm{N}=50$ but not clearly defined for $\mathrm{N}=30$, and 100 .

Moreover, as expected, increasing sample size decreased the MSE. However, this effect varied across conditions. To explain, when the model was correctly specified, increasing the sample sizes at both the first and second level decreased the MSE of the estimates for slope, particularly with increase measurement occasion (i.e., from $t=3$ to 5 decreased MSE estimates from $(0.4082,0.5675,0.6155)$ to $(0.2640,0.4381$, 0.4753 ) respectively across the values of autocorrelation coefficients under FML, Similarly,(from N=30 to 50) MSE decreased from $(0.4082,0.5675,0.6155)$ to $(0.1484,0.1945$, 0.2168 ) under FML, $t=3$, however, this effect varied across conditions under both models. There was an increase or decrease in MSE of the estimates of the fixed effect when either the number of measurement occasion or the number of subjects changed.

The effect of non-autocorrelation assumption violation on the MSE of the estimates of some fixed effect parameters seemed to depend on the autocorrelation level and sample sizes at both levels. In general, the MSE for the estimates of the slope when $\mathrm{t}=5$ were less than when $\mathrm{t}=3$, and that of $\mathrm{t}=10$ less than that of $\mathrm{t}=5$.

\section{DISCUSSION AND CONCLUSION}

When the mean square error (MSE) of the estimators was contrasted across fixed effect estimates, the magnitude of MSE in the parameter estimates depends on the degree of correlation between subjects' data especially, under C-model compared to S-model. MSE of parameter estimates quantify the sum of the variance and squared bias associated with each parameter. For biased outcomes, MSE can be interpreted as a measure of overall accuracy, combining both bias and accuracy of precision into a single value. From the results of our analysis, FML exhibits greater MSE when compared with REML MSE estimates, contrary to prior simulation and theoretical work that demonstrated that for some models, REML, though uniformly less bias than FML may exhibit greater MSE than FML as model complexity increases [15]. A necessary consequence of these facts with respect to the results of our study is that for two equally biased estimates, FML with less MSE, is a measure of efficiency upon which we would expect REML to outperform FML. Overall, the findings of the present study were consistent with those of previous studies with regard to the robustness of multilevel model to the violation of statistical assumptions.

Conclusively, the estimation of level-2 fixed effects of subject characteristics is relatively robust with respect to the violation of non-autocorrelation assumption. This is an important finding, because violating non-autocorrelation assumption led to seriously inaccurate parameter estimates (high MSE values) for the second-level predictors and residuals. The precision of the fixed effects will be decreased with increase heterogeneity caused by autocorrelation which might lead to a widening confidence intervals.

\section{REFERENCES}

[1] Maria, V. G., Hopkins, M., and Stephen W. (2004). Dietary Choline Supplementation Improves Behavioral, Histological, and Neurochemical Outcomes in a Rat Model of Traumatic Brain Injury.

[2] Zeger, S. L., and Harlow, S. D. (1987). Mathematical Models from Laws of Growth to Tolls for Biological Analysis: Fifty Years of Growth. Growth, 51, 1-21. 
[3] Kristopher J. P., Robert C. M., and Nancy E. B. (2008). Latent Growth Curve Modeling. MacCallum University of North Carolina at Chapel Hill Nancy E. Briggs

[4] Kreft, I. G. G., \& de Leeuw, J. (1998). Introducing multilevel modeling. Thousand Oaks, CA: Sage

[5] Woltmam et. al., (2012). Tutorials in Quantitative Methods for Psychology 2012, Vol. 8(1), p. 52-69.

[6] Sullivan, L. M., Dukes, K. A., \& Losina, E. (1999). Tutorial in biostatistics: An introduction to hierarchical linear modeling. Statistics in Medicine, 18, 855-888.

[7] Ramon et. al., (2000). Tutorial in biostatistics modeling covariance structure in the analysis of repeated measures data. statistics in medicine, Statist Med $19: 1793\{1819\}$

[8] Garson David (2013). Hierarchical Linear Modeling: Guide and Applications. SAGE publication inc.

[9] Anya \& Aris (2000). The Linear Regression Model with Autocorrelated Errors: Just Say No to Error
Autocorrelation. Annual Meeting of the American Agricultural Economics Association, July 28-31, 2002.

[10] Raudenbush, S. W. \& Bryk, A. S. (2002). Hierarchical linear models: Applications and data analysis methods (2nd ed.). Thousand Oaks, CA: Sage Publications.

[11] Fitzmaurice et. al., (2011). Applied Longitudinal Analysis, Second Edition, Hoboken, New Jersey: John Wiley \& Sons, Inc.

[12] Gill, J. (2003). Bayesian Methods for the Social and Behavioral Sciences. Chapman \& Hall, New York.

[13] Cohen, J. (1988). Statistical power analysis for the behavioral sciences. New York: LEA.

[14] Maas, C.J.M., \& Hox, J.J. (2005). Sufficient sample sizes for multilevel modeling methodology.

[15] Corbeil, R. R., and Searle, S. R. (1976). Restricted maximum likelihood (REML) estimation of variance components in the mixed model. Technometrics. 